\title{
HIPERDONTIA: RELATO DE CASO COM 10 ELEMENTOS SUPRANUMERÁRIOS
}

Thiago Felippe Oliveira de Macêdo

Maysa Nogueira de Barros Melo

Fabio de Freitas Pereira Freire

Renata Moura Xavier Dantas

Débora Luzia Santos Andrade

Roberto Almeida de Azevedo
Residente de Cirurgia e Traumatologia

Bucomaxilofacialda Universidade Federal da Bahia

/Hospital Santo Antônio - Salvador - Bahia.

Residente de Cirurgia e Traumatologia

Bucomaxilofacialda Universidade Federal da Bahia

/Hospital Santo Antônio - Salvador - Bahia.

Residente de Cirurgia e Traumatologia

Bucomaxilofacialda Universidade Federal da Bahia

/Hospital Santo Antônio - Salvador - Bahia.

Residente de Cirurgia e Traumatologia

Bucomaxilofacialda Universidade Federal da

Bahia/Hospital Santo Antônio - Salvador - Bahia.

Cirurgiã Dentista, aluna do internato de Cirurgia e Traumatologia Bucomaxilofacialda Universidade Federal da Bahia/Hospital Santo Antônio - Salvador - Bahia.

Professor Adjunto do departamento de Cirurgia e Traumatologia Bucomaxilofacialda Universidade Federal da Bahia. /Hospital Santo Antônio - Salvador - Bahia.

Endereço para correspondência: tfelippe@hotmail.com

\begin{abstract}
Resumo
Hiperdontia consiste na alteração do número de unidades dentárias para além do considerado normal na dentição humana. Tais unidades são chamadas de supranumerárias e esse fenômeno tem como etiologias a hereditariedade, o meio ambiente e principalmente algumas alterações sistêmicas. Por este motivo, é considerado raro em pacientes não sindrômicos. $\mathrm{O}$ diagnóstico precoce e adequado tratamento dessa anomalia permite a prevenção de complicações como reabsorção dentária, má oclusão, alteração na erupção e posicionamento de dentes, cistos e/ou tumores. O presente trabalho tem como objetivos relatar um caso raro de dezunidades dentárias supranumerárias e discutir a respeito do mais adequado tratamento.
\end{abstract}

Palavras-chave:Dente supranumerário;Cirurgia bucal; Odontologia.

\section{HYPERDONTIA - A CASE REPORT WITH 10 SUPERNUMERARY TEETH}

\begin{abstract}
Hyperdontia consists on the presence of excessive number of teeth, considering the normal human dentition. These excessive teeth are called supernumeraries and this phenomenon's etiologies are the genetics, the environment and mainly some syndromes. Because of this, it is considered rare at non-syndromic patients. Earlier diagnosis and correct treatment prevent complications like dental resorption, malocclusion, position and eruption teeth's disorders, cysts and/or tumors. The aims of this article are report a rare case of tensupernumerary teeth and discuss its best treatment.
\end{abstract}

Keywords:Supernumerary tooth; Oral surgery;Dentistry. 


\section{INTRODUÇÃO}

A dentição humana considerada normal contém vinte unidades na dentição decídua e trinta e duas na permanente. A alteração para além destes padrões origina as chamadas unidades supranumerárias. Também chamado de hiperdontia, o fenômeno pode ser uni ou bilateral, único ou múltiplo, e possui incidência populacional de 1 a $3 \%$. Sua etiologia é associada a fatores como a hereditariedade, o meio ambiente e principalmente algumas alterações sistêmicas. Algumas teorias buscam explicar a hiperdontia: a teoria da hiperatividade da lâmina dentária na fase de iniciação (que produz novo germe dentário e é considerada a causa mais provável) e a teoria atávica (onde se tem a regressão a padrões de ancestrais primitivos e associação a distúrbios do desenvolvimento). ${ }^{(1,2)}$

A maioria dos dentes supranumerários desenvolve-se durante as duas primeiras décadas de vida. Podem estar presentes em ambas as dentições, todavia são cinco vezes menos freqüentes na decídua. Em relação ao gênero, é irrelevante na primeira dentição, entretanto, na permanente, há uma predileção de 2:1 pelo sexo masculino. Entre os ossos gnáticos, há maior proporção na maxila, com uma forte predileção pela região anterior. Porém, quando múltiplos dentes estão envolvidos (mais de cinco), a região de pré-molar, na mandíbula, é o local mais afetado. ${ }^{(3)}$

Os dentes supranumerários são classificados como mesiodentes, quando ocorrem na linha média, distomolar (região quarto molar), paramolar, com localização lingual ou vestibular a um molar e dentesuplementar. ${ }^{(4)}$ Segundo Azenha, Zorzetto, Marzola,Moura e Handen $^{(5)}$ eles ainda podem ser classificados em rudimentares, quando apresentam diferentes formas e são menores e cônicos ou suplementares quando apresentam morfologia normal.

Os supranumerários múltiplos ocorrem predominantemente na região de pré- molares mandibulares e podem estar associados com algumas síndromes como a de Gardner e displasia cleidocraniana, sendo raro o seu aparecimento como condição isolada. As síndromes de Apert, Crouzon, Down, Ehlers-Danlos,Sturge-Weber, Laband, NanceHoran, HallermannStreiff, entre outras, também são patologias correlacionadas à hiperdontia. ${ }^{(3,6,7)}$

A ocorrência de dentes supranumerários, geralmente, é observada por meio de exame radiográfico de rotina (radiografias panorâmicas e periapicais), sendo de fundamental importância o diagnóstico precoce e tratamento adequado a fim de prevenir conseqüências desfavoráveis associadas a esta anomalia, tais como: impactação de dentes permanentes, 
apinhamento dental, má oclusão, erupção retardada e/ou ectópica, distúrbios na articulação temporomandibular, rotação dentária, formação de diastemas, deslocamentos dentários, reabsorções dentárias, comprometimento de enxertos ósseos alveolares, desarmonia funcional, problemas estéticos e desenvolvimento de patologias orais. ${ }^{(1,8,9,10)}$ Recomenda-se a remoção cirúrgica dos mesmos a fim de evitar futuros transtornos a saúde dos pacientes. ${ }^{(9,10)}$

Um estudo de Corrêa,Ferreira, Friedrich, Pistóia AD ePistóiaGD, ${ }^{(1)}$ constatou a prevalência de 2,5\% de unidades supranumerárias ao avaliar 843 radiografias panorâmicas de pacientes atendidos na Universidade Federal de Santa Maria de janeiro de 1997 a dezembro de 2007 e uma predominância masculina de 2:1.

Candeiro,Praxedes, Bringel, Oliveira, Amorim eFernandes ${ }^{(1)}$ encontraram em seu estudo avaliando 1325 radiografias panorâmicas uma prevalência de 3,54\% também com predileção para o gênero masculino. Leite Segundo, Faria, Silva, Vieira ${ }^{(11)}$ encontraram ao avaliar 1800 radiografias panorâmicas uma prevalência de 1,4\% de dentes supranumerários, porém com maior ocorrência no gênero feminino.

Segundo o estudo de Coelho, Macho, Andrade e Macedo, ${ }^{(8)}$ a prevalência de anomalias dentárias de número demonstram variações entre populações, continentes e raças e está entre 0,6 a $2,7 \%$.

Já estudo de Hamada, Maruo, Araujo, Tanaka, GuarizaFilho, Camargo, ${ }^{(12)}$ demonstrou a prevalência de $2,43 \%$ de unidades supranumerárias com predileção pelo gênero masculino, após avaliar 247 radiografias panorâmicas.

O presente trabalho tem como objetivos relatar um caso raro de dez unidades dentárias supranumerárias em um paciente não sindrômico e discutir a respeito do mais adequado tratamento.

\section{RELATO DE CASO}

Paciente do gênero masculino, 19 anos de idade, melanoderma, foi encaminhado ao serviço de Cirurgia e Traumatologia Bucomaxilofacial da Faculdade de Odontologia da Universidade Federal da Bahia pelo seu ortodontista que observou a presença de unidades dentárias supranumerárias durante a análise de sua documentação ortodôntica. Ao exame clínico do paciente observou-se padrão facial dólicofacial e classe II esquelético, presença de duas unidades dentárias supranumerárias na região das unidades 33 e 43, ausência das 
unidades 35 e 45, apinhamento dentário, oclusão classe III em primeiros molares bilateral e mordida aberta na região de caninos bilateral (Figuras 1 a 5). À radiografia panorâmica observaram-se sinais sugestivos de dez unidades dentárias supranumerárias: oito unidades inclusas (01 na região da unidade 18 e 01 na região da unidade 28 ( $4^{\circ}$ molares), 04 na região da unidade 34 e 02 na região da unidade 44) e dois elementos presentes na cavidade oral na região das unidades 14 e 24 . Solicitou-se tomografia computadorizada da face que mostrou mais detalhadamente o posicionamento das unidades dentárias na arcada dentária. Optou-se por realizar a exodontia das dezunidades supranumerárias, os quatro terceiros molares e as unidades 34 e 44 inclusas sob anestesia geral. Realizou-se anestesia infiltrativa terminal com Lidocaína 2\% com epinefrina1:200.00 em fundo de vestíbulo na região de molares superiores e bloqueio dos nervos alveolar inferior, bucal e mentoniano. Procedeu-se à incisão em envelope na região de superiores e ao longo de toda a arcada inferior, seguida de descolamento mucoperiosteal, osteotomia com broca esférica multilaminada $\mathrm{n}^{\circ} 08$ (Figura4), irrigação com soro físiológico $0,9 \%$ e sutura com Monocryl 4.0. No $10^{\circ}$ dia pós-operatório observou-se a cicatrização completa dos tecidos moles ao exame físico e solicitou-se nova radiografia panorâmica, a qual demonstrou sinais sugestivos de boa cicatrização óssea (Figura $6)$.

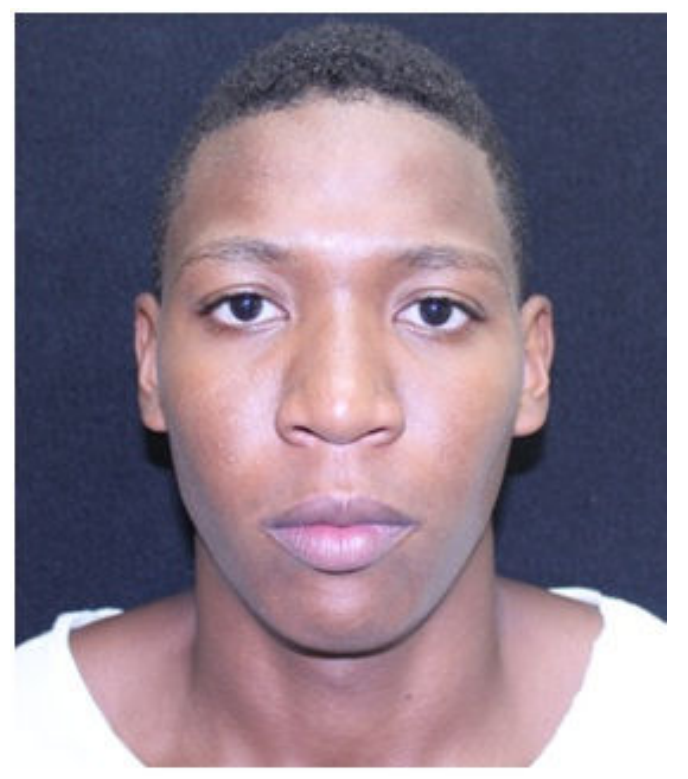

Figura 1 - Norma frontal 


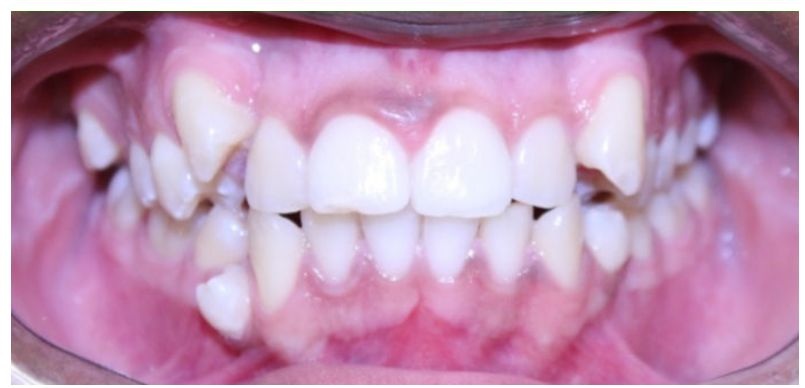

Figura 2-Intra-oral - Norma Frontal

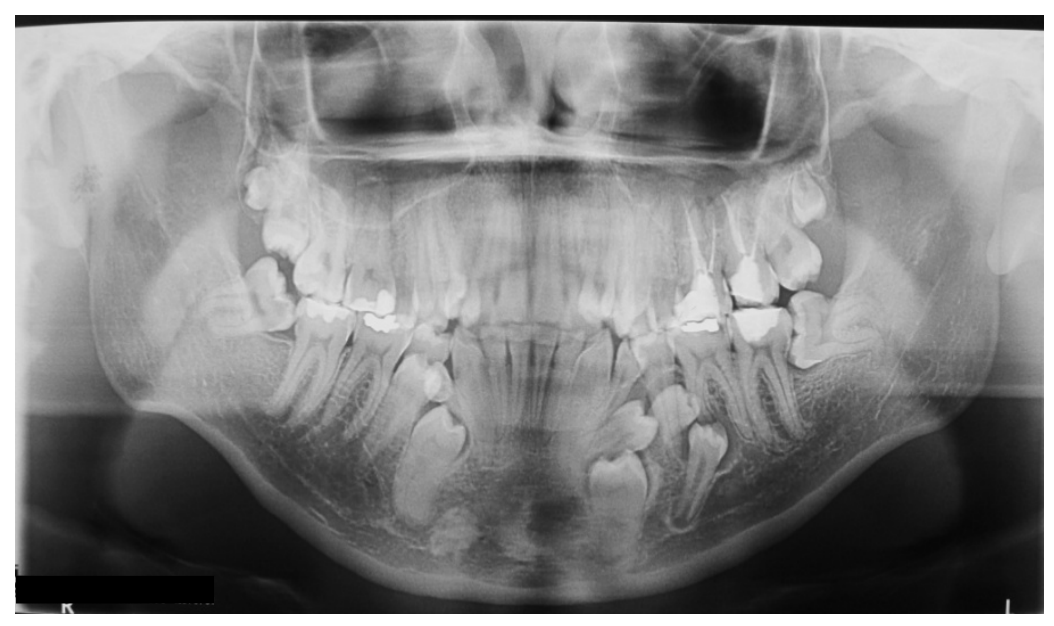

Figura 3- Radiografia Panorâmica dos Maxilares

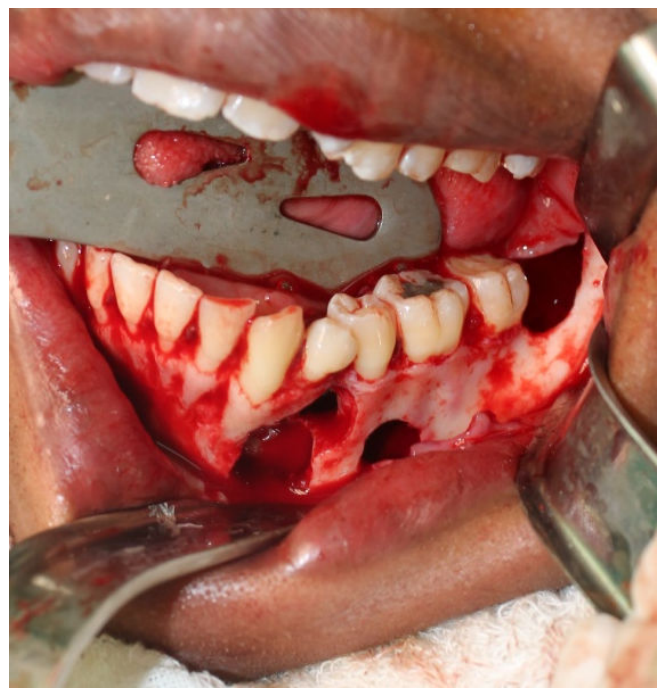

Figura 4-Trans-cirúrgico

Revista Bahiana de Odontologia. 2013 out;4(2):138-146

http://www.bahiana.edu.br/revistas 


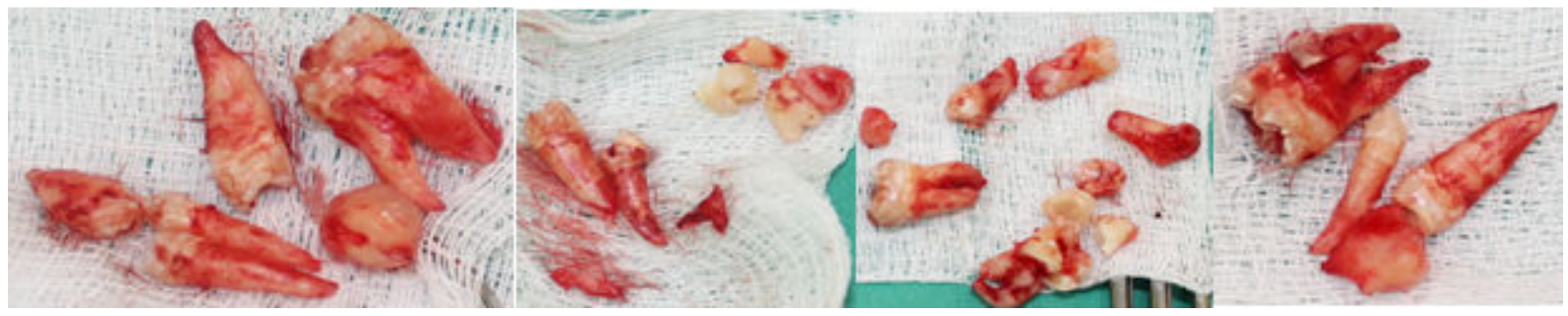

Figura 5 - Unidades extraídas

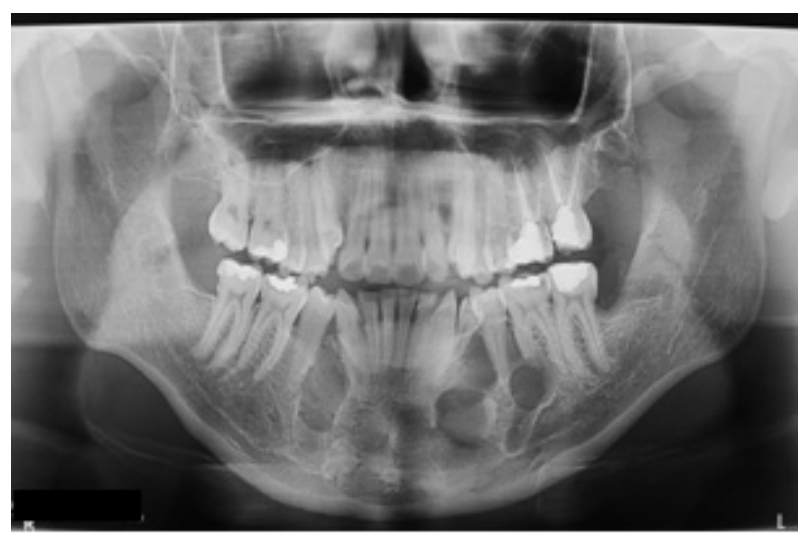

Figura 6- Radiografia Panorâmica Final

\section{DISCUSSÃO}

O presente relato trata-se de um caso raro de hiperdontia por não estar associado a síndrome. A literatura é unânime quanto ao tratamento cirúrgico das hiperdontias, por evitar má-oclusões, distúrbios na articulação temporomandibular, reabsorções dentárias, entre outros.

Segundo Corrêa,Ferreira, Friedrich, Pistóia AD ePistóiaGD, ${ }^{(1)}$ as radiografias panorâmicas são de grande importância para o diagnóstico das unidades dentárias supranumerárias, assim como de outras patologias.

Isso demonstra para Sholapurkar e Pai ${ }^{(2)}$ e Azenha, Zorzetto, Marzola, Moura e Handen ${ }^{(5)}$ a importância no exame clínico e pesquisa radiográfica minuciosa antes de se iniciar qualquer tratamento odontológico. Moruzzi, Raitz, Fenyo, Gambier ${ }^{(13)}$ afirmam que a radiografia panorâmica também deve ser solicitada quando se suspeitar de atrasos na erupção dentária, podendo assim elucidar casos de impactação dentária pela presença de dentes supranumerários. 
Para Almeida, Saavedra Junior, Kawakami, Palis, Mariani, Dottore ${ }^{(10)}$ as tomografias computadorizadas são desnecessárias para a localização destes elementos dentários, visto que as técnicas radiográficas convencionais são suficientes.

Hamada, Maruo, Araujo, Tanaka, GuarizaFilho, Camargo, ${ }^{(12)}$ afirmam que mesmo com uma baixa prevalência, deve-se considerar certa dificuldade no diagnóstico das unidades supranumerárias, especialmente na faixa etária de 11-12 anos, pois existem situações nas quais esses dentes não podem ser visualizados.

No presente relato, o diagnóstico foi conseguido após realização de radiografia panorâmica de rotina durante planejamento ortodôntico e solicitou-se tomografia computadorizada em virtude da grande quantidade de unidades supranumerárias observadas.

Segundo Almeida, Saavedra Junior, Kawakami, Palis,Mariani,Dottore, ${ }^{(10)} \mathrm{e}$ Reis,Giovanini, Namba, Silva e Garcia, ${ }^{(14)}$ o tratamento cirúrgico é unânime na literatura, porém existem algumas controvérsias quanto à época de intervenção cirúrgica, se após a total formação das raízes dentárias ou após preparo psicológico do paciente em casos diagnosticados ainda na infância.

Ainda segundo Reis,Giovanini, Namba, Silva eGarcia, ${ }^{(14)}$ a remoção precoce justificase quando os supranumerários estiverem interferindo no irrompimento ou na formação dos dentes normais adjacentes, prejudicando o desenvolvimento fisiológico da erupção, originando lesões císticas ou tumorais ou irrompendo ectopicamente. Não existindo assim, indicação imediata da remoção cirúrgica de dentes supranumerários na ausência de condições patológicas ou tratamento ortodôntico previsto.

Para Almeida, Saavedra Junior, Kawakami, Palis,Mariani,Dottore, ${ }^{(10)}$ não parece lógico aguardar para realizar as exodontias de supranumerários, pois parece óbvio que na maioria das vezes quanto menor for a raiz menor será a osteotomia e que os riscos de lesionar estruturas nobres são os mesmos independente do momento em que se optar pelo ato operatório.

No caso relatado, optou-se pela remoção após avaliação e planejamento ortodôntico do caso, conduta condizente com a literatura. Não foram observadas complicações em virtude do tratamento estabelecido e o paciente é acompanhado regularmente.

Ao avaliarmos as imagens radiográficas do caso em questão observa-se também semelhança com a maioria dos casos relatados na literatura, pois se trata de dentes suplementares (sem nenhuma alteração na morfologia). Além disso, observou-se como 
complicação da presença de múltiplos dentes supranumerários a alteração do posicionamento normal dos dentes em oclusão.

Segundo Azenha, Zorzetto, Marzola, Moura e Handen ${ }^{(5)}$ alterações como regressão filogenética, como hiperatividade da lâmina dental, trauma sobre o folículo dentário, enfermidades sistêmicas e anomalias de desenvolvimento causam o aparecimento de hiperdontias. O presente caso torna-se por outro lado extremamente raro na literatura, pois não apresenta nenhuma relação com tais alterações e o paciente apresentou acometimento de todos os quadrantes dos maxilares. Não foi encontrada nenhuma característica sindrômica no paciente em questão durante o exame clínico. Ademais, o mesmo não tem conhecimento de familiares portadores de hiperdontia.

É consenso na literatura que a hiperdontia é um fenômeno comum na clínica odontológica e seu diagnóstico precoce é de extrema importância para a decisão de intervenção cirúrgica e o momento que é mais adequado para tal.

\section{CONSIDERAÇÕES FINAIS}

A ocorrência de unidades dentárias supranumerárias é rara em pacientes nãosindrômicos como se observou no caso relatado e pode estar associada a uma série de complicações.

Neste contexto, o diagnóstico precoce e tratamento adequado são de fundamental importância para a obtenção de estética, oclusão e função dentárias satisfatórias.

O tratamento é cirúrgico e não existe consenso na literatura a respeito do melhor momento para realizá-lo. Portanto, maiores estudos devem ser realizados para o estabelecimento de protocolos para intervenção na presença de dentes supranumerários.

\section{REFERÊNCIAS}

1 Corrêa FG, Ferreira FV, Friedrich LR, Pistóia AD, Pistóia GD. Prevalência de dentes supranumerários- estudo retrospectivo. Int J Dent. 2009;8(1):11-15.

2Sholapurkar AA, Pai KM. Erupted supernumerary teeth unusual positions. Rev ClínPesqOdontol. 2010;6(1):11-13.

3 Neville BW, Damm DD, Allen CM, Bouquot JE. Patologia oral e maxilofacial. $2^{\mathrm{a}}$ ed. Rio de Janeiro: Elsevier; 2009. p 68-72. 


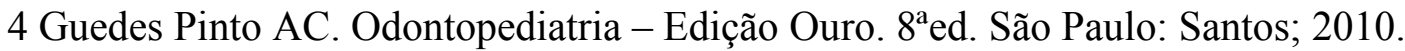

5 Azenha MR, Zorzetto DLG, Marzola C, Moura LA, Handem RH. Abordagem cirúrgica de dente supranumerário (Mesiodens) na região palatina: caso clínico.

RevPortEstomatolCirMaxilofac2007;48:37-41.

6Buggenhout GV, Bailleul FI. Mesiodens. Eur J MedGenet. 2008;51:178-81.

7Yagüe GJ, Berini AL, Gay EC. Multiple supernumerary teeth not associated with complex syndromes: a retrospective study. Med Oral Patol Oral Cir Bucal. 2009;14(7):331-6.

8 Coelho A, Macho V, Andrade D, Macedo P, Areias C. Prevalência e distribuição de dentes supranumerários numa população pediátrica. Um estudo

radiográfico.RevPortEstomatolMedDentCirMaxilofac. 2011;52(4):189-192.

9Candeiro GTM, Praxedes ACS, Bringel AFS, Oliveira FRR, Amorim HHT, Fernandes LA. Prevalência de dentes supranumerários em radiografias panorâmicas numa amostra populacional do Ceará. Rev ABRO 2009;11(3):47-50.

10 Almeida TE, Saavedra Junior J, Kawakami PY,Palis CA, Mariani PB, Dottore AM. Hiperdontia: Relato de caso com 8 elementos supranumerários. Rev. odontol. Univ. Cid. São Paulo 2010;22(1):78-84.

11 Segundo AVL, Faria DLB, Silva UH, Vieira ITA. Estudo epidemiológico de dentes supranumerários diagnosticados pela radiografia panorâmica. Rev. cir. traumatol. bucomaxilo-fac.2006;6(2):53-56.

12Hamada MH, Maruo IT, Araujo CM, Tanaka OM, GuarizaFilho O, Camargo ES.Prevalência de dentes supranumerários em pacientesque procuraram tratamento ortodôntico.Arch Oral Res. 2011;7(2):141-6.

13Moruzzi LPD, Raitz R, Fenyo PM, Gambier VCR. Quarto e quinto molares em maxila: Relato de um raro caso clínico. Rev. bras.ciênc.saúde. 2005;3(6):13-17.

14 Reis, LFG, Giovanini A, Namba, EL, Silva, ELFM, Garcia, MA.Dentes supranumerários retidos interferindo no tratamento ortodôntico. RSBO. 2006;3(2):20-25. 